nhân nghiên cứu, chiếm tỷ lệ 3,9\%. Tỷ lệ này cũng phù hợp với các nghiên cứu khác trong nước và trên thế giới.Tỷ lệ đột biến gen NRAS trong nghiên cứu của Peeters và cộng sự (2015) là 7,4\%[4]. Ở Việt Nam, Vũ Thị Nhung và Nguyễn Thuận Lợi (2018) báo cáo tỷ lệ đột biến gen NRAS là 4,1\% [8].

Trong nghiên cứu này, chúng tôi không tìm được sự liên quan giữa tình trạng đột biến gen NRAS với giới tính, vị trí khối u nguyên phát, nồng độ CEA huyết thanh và độ mô học khối u.

Đột biến gen BRAF. Trong số 76 bệnh nhân tham gia nghiên cứu, có 7 bệnh nhân đột biến gen $B R A F$, chiếm tỷ lệ $9,2 \%$. Các nghiển cứu khác cũng cho kết quả tương tự. Tỷ lệ đột biến BRAF trong các nghiên cứu COIN, CAIRO2, NORDIC VII, CRYSTAL, PRIME trong khoảng 6$12 \%$ [4]. Ớ trong nước, Thị Nhung và Nguyễn Thuận Lợi (2018) thông báo tỷ lệ đột biến gen BRAF là 6,9\% [8].

Đột biến BRAF có mối liên quan với u ở đại tràng phải, số lượng tạng di căn lớn, u kém biệt hóa, giới tính nữ [4]. Trong nghiên cứu này, chúng tôi nhận thấy đột biến gen BRAF hay gặp ở các bệnh nhân có nồng độ CEA huyết thanh tăng trên $20 \mathrm{ng} / \mathrm{ml}$ tại thời điểm chẩn đoán.

\section{KẾT LUÂNN}

Nghiên cứu 76 trường hợp ung thư đại trực tràng di căn chúng tôi ghi nhận tỷ lệ đột biến KRAS, NRAS và BRAF lần lượt là $44,7 \% ; 3,9 \%$ và 9,2\%. Tỷ lệ đột biến KRAS ở các bệnh nhân có nông độ CEA huyết thanh tại thời điểm chẩn đoán > 5ng/ml cao hơn có ý nghĩa thống kê so với các bệnh nhân có nồng độ CEA huyết thanh tại thời điểm chẩn đoán $\leq 5 \mathrm{ng} / \mathrm{ml}$. Chúng tôi cũng quan sát thấy tỷ lệ đột biến BRAF ở các bệnh nhân có nồng độ CEA huyết thanh tại thời điểm chẩn đoán > 20ng/ml cao hơn có ý nghĩa thống kê so với các bênh nhân có nồng đô CEA huyết thanh tại thời điểm chẩn đoán $\leq 20 \mathrm{ng} / \mathrm{ml}$.

\section{TÀI LIÊU THAM KHẢO}

1. H. Sung, J. Ferlay và R. L. Siegel (2021) Global Cancer Statistics 2020: GLOBOCAN Estimates of Incidence and Mortality Worldwide for 36 Cancers in 185 Countries. 71(3), 209-249.

2. E. Van Cutsem, A. Cervantes, B. Nordlinger và cộng sự (2014). Metastatic colorectal cancer: EŚMO Clinical Practice Guidelines for diagnosis, treatment and follow-up. Ann Oncol, 25 Suppl 3, iii1-9.

3. A. M. Krasinskas (2011). EGFR Signaling in Colorectal Carcinoma. Pathology Research International, $2011,932932$.

4. J. Gong, M. Cho và M. Fakih (2016). RAS and BRAF in metastatic colorectal cancer management. J Gastrointest Oncol, 7(5), 687-704.

5. W. A. Messersmith (2019). NCCN Guidelines Updates: Management of Metastatic Colorectal Cancer. J Natl Compr Canc Netw, 17(5.5), 599-601.

6. F. Selcukbiricik, A. Bilici, D. Tural và công sự (2013). Are high initial CEA and CA 19-9 levels associated with the presence of K-ras mutation in patients with metastatic colorectal cancer? Tumour Biol, 34(4), 2233-2239.

7. B. A. Tuyết và N. V. Hiếu (2017). Nghiên cứu một số đặc điểm lâm sàng, cận lâm sàng và tình trạng đột biến gen KRAS trong ung thư đại trực tràng tại Bệnh viện $K$, Luận văn Nghiên cứu sinh ung thư', Trường Đai hoc Y Hà Nôi.

8. V. T. Nhung, N. T. Lợi và H. T. Nhung (2018). Nhân xét tinh trang đột biến gen KRAS, NRAS BRÁF trên bệnh nhân ung thư đại trực tràng tại Bệnh viện Bạch Mai, Khóa luận tốt nghiệp đại học ngành y khoa, Đại học Quốc gia Hà Nội.

\title{
TÁCH LỚP LIPID MẪU CHUẨN TRONG DUNG DICH Có NỒNG Độ MUỐI CAO BẰNG SẮC KÝ LỚP MỎNG VÀ CHIẾT PHA RẮN
}

Trịnh Minh Việt*, Nguyễn Quốc Chiến*, Đỗ Tuấn Mến*

\section{TÓM TẮT}

Các quy trình TLC, SPE đã công bố có thể tách tốt các phân lớp lipid từ mẫu sinh học trong tự nhiên như (lipid trong thực phẩm, lipid trong huyết thanh người...). Chúng tôi, đã ứng dụng phân tích lipid trong dung dịch có nồng độ muối kiềm cao (DDM: dung dịch

\footnotetext{
*Viện 69, Bộ tư lệnh Bảo vệ Lăng Chủ tịch Hồ Chí Minh. Chịu trách nhiệm chính: Trịnh Minh Việt

Email: dr.minhviet@gmail.com

Ngày nhận bài: 16.6 .2021

Ngày phản biên khoa họ: 12.8.2021

Ngày duyệt bài: 18.8.2021
}

bảo quản mô sinh học), bằng các quy trình TLC, SPE này; tuy nhiên quy trình $S P E$ theo Kaluzny và Agren không lặp lại được đối với mấu lipid chiết từ DDM, chưa thu được kểt quả như tác giả công bố; Các kết quả TLC thu được phù hợp với một số công bố đã được chấp nhận rộng rãi, song rất khó nhận diện cholesterol với DG trong kết quả phân tích mâuu hổn hợp. Đây rõ ràng là điểm hạn chế nếu thành phần đích là DG và cholesterol. Qua khảo sát, Chúng đã cải tiến TLC (Viện 69) một bước với hỗn hợp dung môi B (C6-DEE-methanol-acid acetic; 90:20:3:2) do đơn giản và tách khá tốt các lớp lipid chuẩn đơn cũng như hỗn hợp. Trường hợp cân tách thêm các thành phần MG, DG có thể khai triển thêm một bước với hỗn hợp chứa 
dung môi phân cực, chẳng hạn chúng tôi đã thu được kết quả có cải thiện khi khai triển thêm một bước bằng $C_{6}-D E E-a c e t o n ~(60: 40: 5)$ đến $12 \mathrm{~cm}$. Mục tiêu: Đánh giá hiệu quả tách thành phân lipid trong dung dịch muối kiềm bằng phương pháp chiết pha rắn và sắc ký lớp mỏng, lựa chọn, tối ưu quy trình ứng dụng phân tích lipid trong dung dịch muối. Đối tượng và phương pháp: Tiến hành trên 6 mẫu chuẩn lipid gồm: FFA (C12:0 - C22:0; Sigma); các acyl glycerol gắn từ 1 đến 3 acid béo (C14:0 - C18:0) (Sigma); hỗn hợp 37 FAMEs (Supelco), hỗn hợp phospholipid PL gồm $P C$ và $P E ; 6$ mâu lipid chuẩn, 6 mấu lipid mô mõ bảo quản trong dung dịch muối (DDM) được thực hiện lặp lại $n=6$ cho mỗi mẫu. Quy trình TLC sửa đổi tại Viện 69. Phương pháp nghiên cứu mô tả tiến cứu trong thực nghiệm. Kết quả: Với phương pháp của Viện 69 cho kết quả ổn định, độ lặp lại cao và tách tốt các lớp lipip : Phospholipid; monoglycerid; diglycerid; cholesterol; acid béo tự do; triglycerid; cholesterol esthercó Rf lần lượt là : 0; $(0,0-0,03) ;(0,11-0,28)$; $(0,12-0,20) ;(0,33-0,52) ;(0,66-0,75) ;(0,80-$ $0,90)$. Các lớp lipid từ bản TLC cũng thu lại được khá đơn giản cho phân tích tiếp. Cho phép áp dụng tốt để tách lớp các lipid trong DDM tại Viện 69. Kêt luận: Ứng dụng phương pháp TLC sửa đổi tại Viện 69 có thể tách các lớp lipid hòa tan trong DDM một cách hiêu quả, đây là một bước quan trọng trong chuẩn bị mẫu cho các phân tích lipid chuyên sâu bằng sắc ký khí, sắc ký lỏng hiệu năng cao

Tư khóa: sắc ký lớp mỏng (TLC), chiết pha rắn (SPE), lipid, dung dịch muối (DDM).

\section{SUMMARY}

\section{SEPARATION OF THE STANDARD SAMPLE LIPID LAYER IN A HIGH CONCENTRATION SALT SOLUTION BY THIN-LAYER- CHROMATOGRAPHY AND SOLID-PHASE- EXTRACTION}

The published TLC, SPE processes can well separate lipid subclasses from biological samples in nature such as (lipids in food, lipids in the human serum...). We have applied lipid analysis in solutions with high alkaline salt concentration (DDM: biological tissue preservation solution), by these TLC, SPE processes; however, the SPE procedure according to Kaluzny and Agren was not repeatable for lipid samples extracted from DDM, the results were not obtained as announced by the author; The obtained TLC results are in agreement with a number of widely accepted publications, but it is difficult to identify cholesterol with DG in the mixed sample analysis results. This is clearly a limitation if the target ingredients are DG and cholesterol. Through the survey, we improved TLC (Institute 69) one step with solvent mixture B (C6-DEE-methanol-acetic acid; 90:20:3:2) due to its simplicity and good separation of standard lipid layers. single as well as mixed. In case it is necessary to separate more MG components, DG can go one step further with a mixture containing polar solvents, we have obtained improved results when expanding one step further with C6-DEEacetone $(60: 40: 5)$ to $12 \mathrm{~cm}$. Objective: To evaluate the efficiency of separating lipid components in alkaline salt solution by solid-phase extraction and thin layer chromatography, to select and optimize the application process of lipid analysis in saline solution. Subjects and methods: Conducted on 6 standard lipid samples including FFA (C12:0 - C22:0; Sigma); glycerol acyl with 1 to 3 fatty acids (C14:0 - C18:0) (Sigma); a mixture of 37 FAMEs (Supelco), a mixture of PL phospholipids including PC and PE; 6 standard lipid samples, 6 adipose tissue lipid samples preserved in saline solution (DDM) were repeated $n=6$ for each sample.Revised TLC Process at Institute 69. Experimental descriptive research method. Results: With the method of Institute 69 for stable results, high repeatability and good separation of lipid layers: Phospholipid; monoglycerides; diglycerides; cholesterol; free fatty acids; triglycerides; cholesterol esther has Rf respectively: 0; $(0.0-0.03) ;(0.11-$ $0.28) ;(0.12-0.20) ;(0.33-0.52) ;(0.66-0.75) ;(0.80$ - 0.90). The lipid layers from the TLC plate were also obtained quite simply for further analysis. Allows good application for separation of lipids in DDM at Institute 69. Conclusion: Application of the modified TLC method at Institute 69 can effectively separate the soluble lipid layers in DDM, which is an important step in sample preparation for intensive lipid analyzes by gas chromatography, high-performance liquid chromatography

Keywords: thin layer chromatography (TLC), solid -phase extraction (SPE), lipid, saline solution (DDM).

\section{I. ĐẶT VẤN ĐỀ}

Nghiên cứu thành phần lipid một cách tổng thể là xu hướng hiện nay do giá trị của nó trong nhiều lĩnh vực và cho các mục đích khác nhau. Ớ xu hướng này, hiện chủ yếu là trong các hệ thống chuyển hóa sinh học thuộc các lĩnh vực y, dược. Nghiên cứu trong các nguồn mẫu mà ở đó lipid tự biến đổi hóa học - chẳng hạn trong dung dịch muối kiềm nồng độ cao (DDM), còn chưa nhiều. Trước đây chúng tôi đã phân tích thành phần tổng thể của lipid chiết từ loại DDM này cả bằng sắc ký khí và sắc ký lỏng. Kết quả cho thấy còn một số thành phần chưa thể nhận diện được trên các sắc đồ do chồng lấn và có thể cả do bị biến đổi,trong đó có các lớp lipid quan trọng như cholesterol, phospholipid.

Để khắc phục vấn đề này, chúng tôi tiến hành tách sơ bộ trước các lớp lipid chiết từ mẫu DDM bằng SPE. SPE có nhiều ưu điểm như nhanh, đơn giản, thu mẫu thuận tiện... và được cho là có thể thay thế cả cho chiết lỏng - lỏng, TLC hay thậm chí thay thế một phần cho HPLC. Tuy vậy, các quy trình SPE đã công bố thường gồm không ít bước và cũng đòi hỏi độ chính xác khá cao trong thao tác thủ công ở phòng thí nghiệm. Ngoài ra dù lipid đã được chiết tách riêng, song chúng tôi thấy các đặc điểm riêng của nguồn mẫu cũng có ảnh hưởng đến kết quả phân tích. Vì vậy cần xác thực lại các quy trình về khả năng 
áp dụng cụ thể. Chúng tôi cũng kiểm tra lại một số quy trình $T L C$ cho chuẩn bị mẫu nói trên, đồng thời để đánh giá hiệu quả của SPE để tách chiết các lớp lipid bị hòa tan vào trong DDM.

\section{II. ĐỐI TƯợNG VÀ PHƯƠNG PHÁP NGHIÊN CỨU}

2.1. Đối tượng. Tiến hành trên 6 mẫu chuẩn lipid gồm: FFA (C12:0 - C22:0; Sigma); các acyl glycerol gắn từ 1 đến 3 acid béo (C14:0 - C18:0) (Sigma); hỗn hợp 37 FAMEs (Supelco), hỗn hợp phospholipid $\mathrm{PL}$ gồm $\mathrm{PC}$ và $\mathrm{PE} ; 8$ mẫu lipid chuẩn, 6 mẫu lipid mô mõ bảo quản trong dung dịch muối (DDM) được thực hiện lặp lại $n=6$ cho mỗi mẫu.

Thời gian tiến hành từ tháng 6 năm 2019 đến tháng 6 năm 2021

\subsection{Vật liệu nghiên cứu}

Mẫu nghiên cứu là các dung dịch chuyên dùng có nồng độ muối cao (DDM), bao gồm:

- DDM pha mới từ hóa chất tinh khiết; DDM đã sử dụng để bảo quảnmẫu mô sinh học.

- Quy trình tách lớp lipid bằng SPE theo Kaluzny [4] và Agren [3];

- Quy trình tách lớp lipid bằng TLC nhiều bước (MOD-TLC);

- Quy trình tách lipid bằng TLC 1 bước (1 hỗn hợp dung môi).

Các dung môi tinh khiết sắc ký gồm: diethyl ether (DEE), n-pentan, n-hexan $\left(\mathrm{C}_{6}\right)$, dichloromethane, chloroform, methanol, aceton, acetonitril (Merck). Các chất chuẩn tinh khiết sắc ký gồm: các acid béo tự do dạng đông khô (C12:0 - C22:0; Sigma); các acyl glycerol gắn từ 1 đến 3 acid béo mạch trung bình (C14:0 C18:0) dạng đông khô (Sigma); hỗn hợp 37 acid béo dạng methyl esther trong dichloro methane (Supelco), methyl heptadecanoat; hỗn hợp phospholipid (PL) gồm phosphatidyl cholin (PC) và ethanolamin $(\mathrm{PE})$

Các hóa chất khác đạt mức tinh khiết phân tích, gồm: khí nitơ sạch $(99,99 \%$ ), khí heli 99,999 \%, khí hydro 99,99\%, natri sulphat khan, acid acetic băng, iod tinh thê..

Bản TLC silicagel F60 (Merck), bể TLC thủy tinh, các dụng cụ thủy tinh trang bị trong phòng thí nghiệm.Cột SPE silica aminopropyl BondElut (Harbour City, CA, USA);Buồng giải SPE gắn bơm hút chân không. Máy GC (Thermo Sci., Mỹ) trang bị cột cột VF-5ht (Agilent, $30 \mathrm{~m} \times$ 0,32 mm ID, chiều dày pha tĩnh $0,15 \mu \mathrm{m})$, và đầu dò ion hóa ngọn lửa.

\subsection{Phương pháp nghiên cứu}

- Thiết kế nghiên cứu: nghiên cứu mô tả, tiến cứu trong thực nghiệm.
- Phương pháp: Lăp lại các quy trình SPE, TLC đã công bố đầy đủ để tách lớp lipid chiết từ các mẫu DDM. Các lipid chuẩn được pha định lượng vào $\mathrm{DDM}$, để ở phòng thí nghiệm trong 1 tháng sau đó chiết lại để làm mẫu phân tích theo các quy trình khảo sát. Đối chiếu với các lipid chuẩn phân tích trực tiếp để đánh giá ảnh hưởng của DDM. Mẫu lipid sau khi tách sơ bộ bằng TLC cũng như SPE được kiểm tra thêm bằng sắc ký khí (GC).

Lựa chọn quy trình phù hợp để phân tích lipid chiết từ mẫu DDM đã dùng để ướp bảo quản mẫu mô sinh hoc.

\subsection{Các bước tiến hành}

2.3.1. Chuẩn bị mẫu: Pha vào $1 \mathrm{ml}$ clorofrom các lipid chuẩn thương phẩm gồm: $P C, P E$, triheptadecanoin, cholesteryl octadecanoate, heneicosanoate; acid palmytic, acid stearic, acid oleic và a-oleic (mỗi thành phần $100 \mu \mathrm{g}$ ). Dùng dung dịch lipid chuẩn này để cho thêm vào DDM theo thể tích.

2.3.2. Chiết lipid từ DDM: Chiết lipid trong mẫu DDM theo phương pháp chiết lỏng - lỏng bằng hỗn hợp $D E E / C_{6}(3: 1 \mathrm{v} / \mathrm{v})$, xác định hàm lượng lipid chiết được bằng phương pháp cân.

2.3.3. Tách các lớp lipid bằng TLC

- Tách theo quy trình sử dụng 6 loại hỗn hợp dung môi của Ruiz \& Ochoa [6];

- Tách theo quy trình sử dụng 3 loại hỗn hợp dung môi của White và cộng sự [7];

- Tách theo quy trình sữ dụng 2 loại hỗn hợp dung môi của Kupke [4];

- Tách theo hai quy trình sử dung 1 hỗn hợp

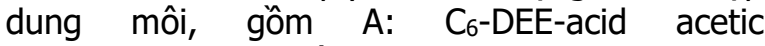
$(80: 15: 1, v / v)[1] ;$ và $B-C_{6}$-DEE-methanol-acid acetic (90:20:3:2, v/v) [2].

Hiên màu lipid trên bản TLC bằng hơi iod ở $50^{\circ} \mathrm{C}$, sau đó thu riêng các đốm để kiểm tra bằng sắc ký khí (GC).

2.3.4. Tách các lớp lipid bằng SPE. Sử dung côt SPE-aminopropyl tách các mẫu lipid chiết theo quy trình của Agren [3] và Kaluzny [4]. Kiểm tra các phân đoạn bằng TLC và GC.

2.3.5. Phân tích kiếm tra các phân đoạn lipid bằng sắc ký khí. Phân tích các phân đoan lipidđã tách bằng SPE và TLC trên cột VF-5ht theo phương án bơm mẫu trực tiếp vào cột (cold on-column), giải theo chương trình nhiệt độ từ $50^{\circ} \mathrm{C}$ (giữ 1 phút), tăng $16^{\circ} \mathrm{C} /$ phút lên $170^{\circ} \mathrm{C}$, rồi tăng tiếp $7{ }^{\circ} \mathrm{C} /$ phút lên $330^{\circ} \mathrm{C}$ sau đó tăng $16^{\circ} \mathrm{C} /$ phút lên $370^{\circ} \mathrm{C}$, giữ 10 phút. Tốc độ khí mang (heli) $2,2 \mathrm{ml} /$ phút. Ghi sắc đồ bằng đầu dò ion hóa ngọn lửa ở nhiệt độ $375^{\circ} \mathrm{C}$. Khí đốt dùng 
hỗn hợp hydro/không khí/nitơ theo tỷ lệ 30/350/30 (ml/phút).

\section{KẾT QUẢ NGHIÊN CứU VÀ BÀN LUÂ̂N}

3.1. Đặc điểm và hiệu quả tách các lớp lipid trong dung dịch muối bằng sắc ký lớp mỏng. Ở nghiên cứu này, chúng tôi sử dụng TLC một chiêu với một số hỗn hợp dung môi tham khảo, gồm cả khai triển một bước và nhiều bước (MOD-TLC) để đánh giá đặc điểm, hiệu quả tách các lớp lipid trong mẫu. Kết quả tách các mẫu lipid chuẩn trước cũng như sau khi cho vào DDM và chiết lại không có khác biệt, như minh họa ở hình 1.

Theo quy trình MOD-TLC của cả 3 tác giả trên, các lớp lipid chuẩn trong mấu tách được khá rõ. Thứ tự tách ra trên bản TLC của các lipid giống nhau theo hướng khai triển dung môi, song nằm ở các vị trí ( $\mathrm{R}_{\mathrm{f}}$ ) có chút khác nhau. Ở mẫu hỗn hợp lipid chuẩn, quy trình của Ruiz cho hiệu quả tách tốt hơn, song DG và cholesterol cũng chưa tách thật tốt như 2 quy trình còn lại.

Các phương pháp khai triển một bước đều tách được các lipid trong mẫu chuẩn (hình 2). Thứ tự tách ra của các lớp theo hướng khai triển giống như quy trình của các tác giả nước ngoài. Một số đặc điểm tách có khác ở hai phương pháp sau gồm: MG di chuyển rất ít (tương tự phương pháp của Ruiz), CE di chuyển nhiều hơn. Các đặc điểm này phù hợp với tính chất của hỗn hợp dung môi sử dụng.

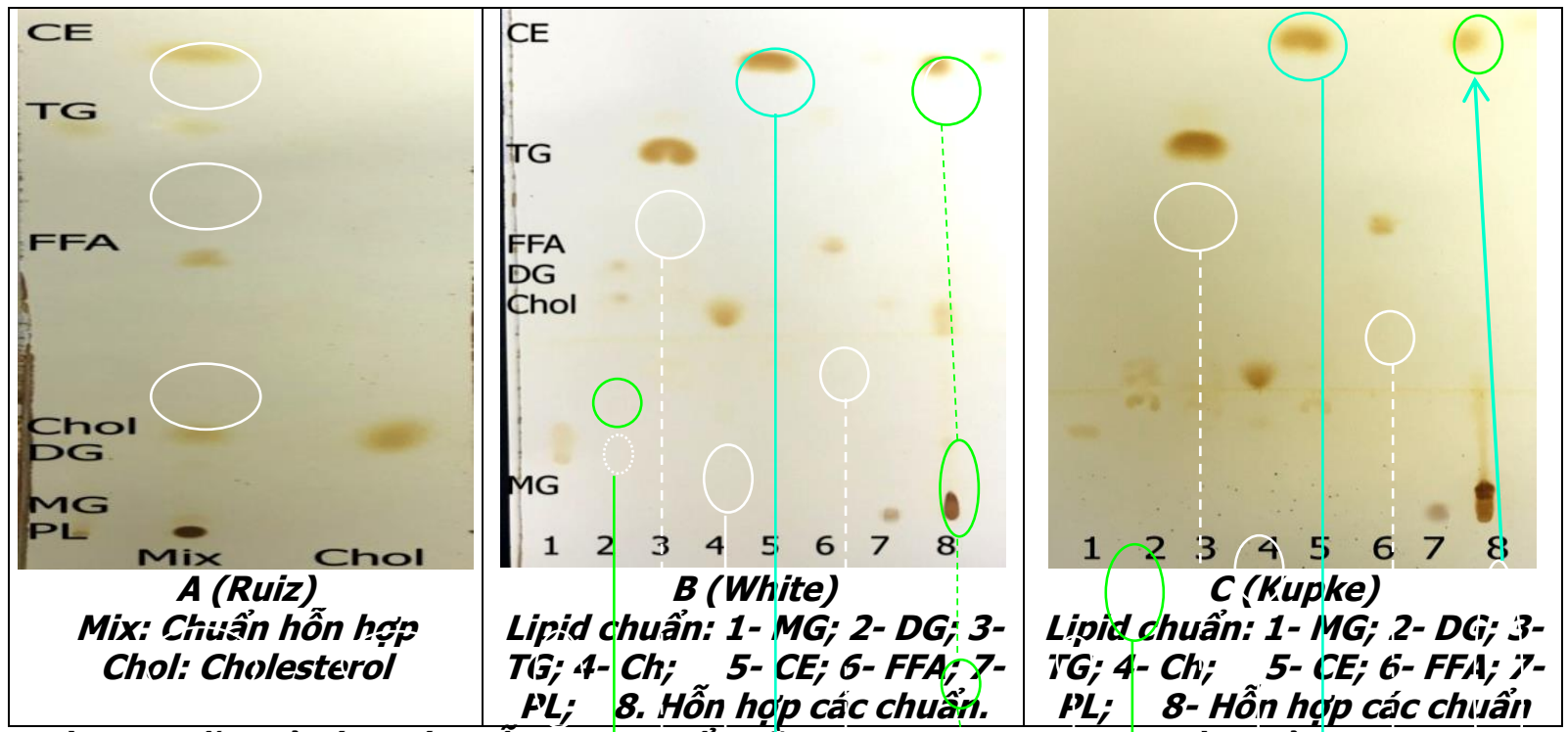

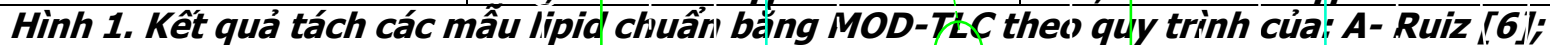
B-White ["7]; và Kuphise [4t].

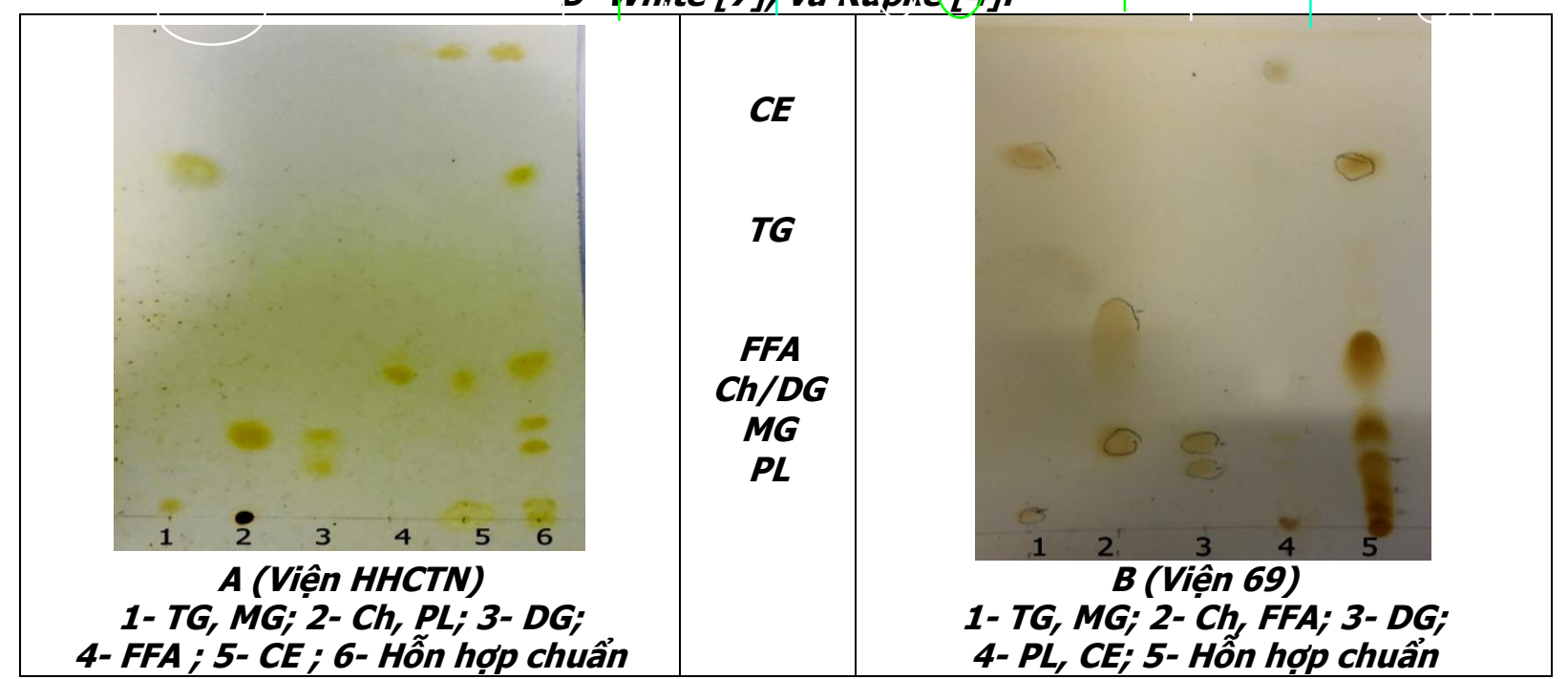


Hình 2. Kết quả tách các mẫu lipid chuẩn bằng TLC theo quy trình khai triển một bước với hỗn hợp dung môi A (Viện Hóa học các hợp chât thiên nhiên - HHCTN) [1]; và B (Viện 69) [2].

ở hai quy trình khai triển một bước, DG cũng tách thành 2 đốm gân nhau như trong các quy trình nhiều bước, tương ứng với các dạng 1,2- và 1,3-DG. Và cũng rất khó phân biệt cholesterol với DG trong kết quả phân tích mẫu hốn hợp. Đây rõ ràng là điểm hạn chế nếu thành phần đích là DG và/hoặc cholesterol. Độ di chuyển của các lớp lipid trên bản TLC khai triển theo các phương pháp khác nhau tính toán được như ở bảng 1.

Bảng 1. Độ di chuyển (Rf) của các lipid theo các phương pháp tách bằng TLC

\begin{tabular}{|c|c|c|c|c|c|}
\hline \multirow{2}{*}{ Lớp Lipid } & \multicolumn{5}{|c|}{ Giá tri Rf theo phương pháp/tác giả } \\
\cline { 2 - 6 } & Ruiz & White & Kupke & Viện HHCTN & Viện $\mathbf{6 9}$ \\
\hline Phospholipid & 0 & 0 & 0 & 0 & 0 \\
\hline Monoglycerid & 0,02 & 0,13 & 0,10 & 0,01 & $0,0-0,03$ \\
\hline Diglycerid & $0,06-0,31$ & $0,31-0,38$ & $0,11-0,13$ & $0,07-0,11$ & $0,11-0,28$ \\
\hline Cholesterol & 0,31 & 0,30 & 0,13 & 0,11 & $0,12-0,20$ \\
\hline Acid béo tự do & 0,44 & 0,44 & 0,27 & 0,21 & $0,33-0,52$ \\
\hline Triglycerid & 0,50 & 0,63 & 0,40 & 0,50 & $0,66-0,75$ \\
\hline Cholesterol esther & 0,56 & 0,75 & 0,50 & 0,64 & $0,80-0,90$ \\
\hline
\end{tabular}

Độ di chuyển của các lớp lipid trên bản TLC giữa các lần phân tích không có sai lệch lớn. Số liệu thu được cho thấy, các chế độ khai triển cũ̃ng như các hốn hợp dung môi đã khảo sát đều tách được các lớp lipid theo thứ tự như nhau. Các quy trình MOD-TLC cho kết quả ổn định vàcó tốt hơn, có thể do hiệu quả của việc khai triển dung môi nhiều lần qua phần dưới của bản gel.

Kết quả TLC thu được phù hợp với một số công bố đã được chấp nhận rộng rãi. Theo đó pha động phổ biến là hexan-diethyl ether-acetic (70:30:1) cho phép tách tốt các glycerid và FFA. Cholesterol và $\mathrm{PL}$ là những thành phần được quan tâm trong rất nhiều lĩnh vực. Mặc dù $\mathrm{GC}$ và sắc ký lỏng hiệu năng cao (HPLC) có thể tách tốt tất cả các lớp lipid (hình 5), song rất khó nhận diện cholesterol và PL- thường có lượng không lớn, trong các mẫu chứa nhiều glycerid.

Qua khảo sát, chúng tôi lựa chọn quy trình TLC một bước với hỗn hợp dung môi $B\left(\mathrm{C}_{6}\right.$-DEEmethanol-acid acetic; 90:20:3:2) do đơn giản và tách khá tốt các lớp lipid chuẩn đớn cũng như hỗn hợp. Trường hợp cần tách thêm các thành phần MG, DG có thể khai triển thêm một bước với hỗn hợp chứa dung môi phân cực, chẳng hạn chúng tôi đã thu được kết quả có cải thiện khi khai triển thêm một bước bằng $\mathrm{C}_{6}$-DEE-aceton (60:40:5) đến $12 \mathrm{~cm}$.

3.2. Đánh giá đặc điểm và hiệu quả tách lipid bằng chiết pha rắn

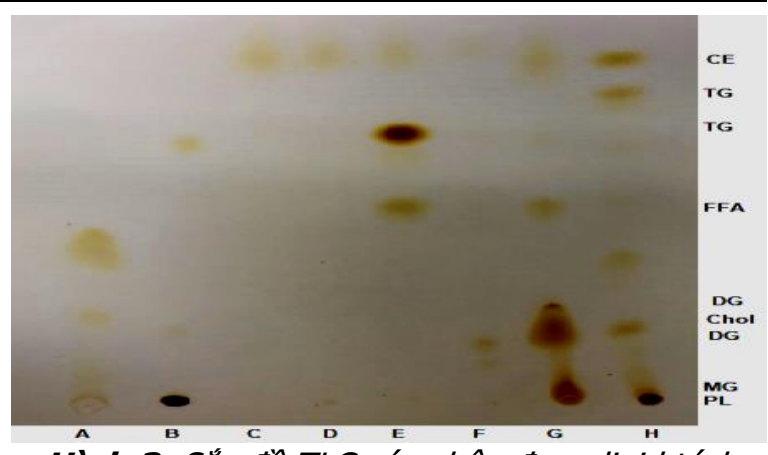

Hình 3. Sắc đồ TLC các phân đoạn lipid tách theo quy trinh SPE của Kaluzny.

Các dải tương ứng các phân đoạn: $A-$ các lipid không phân cực; B- acid béo tự do (FFA); Cphospholipid (PL); D- cholesterol este (CE); Etriglycerid (TG); F- cholesterol; G- diglycerid (DG); H- monoglycerid (MG).

Kiểm tra bằng TLC (hình 3 ) cho thấy, quy trình SPE theo Kaluzny chưa thu được kết quả như tác giả công bố. Trong các phân đoạn vẫn còn lẫn các lớp lipid khác. Chúng tôi chưa tìm ra nguyên nhân của việc không lặp lại được quy trình này, có thể do các lipid chuẩn chịu ảnh hưởng của DDM và thay đổi tương tác với pha tĩnh trong cột SPE. Chỉ riêng phân đoạn $C$ tách được phần lớn $\mathrm{PL}$ với độ tinh sạch tốt. SongPL còn được giải ra khá nhiều ở phân đoạn $A$ và một phần nhỏ ở phân đoạn $\mathrm{D}$. Chúng tôi thấy quy trình SPE này khó sử dụng để thay thế chiết lỏng lỏng trong chuẩn bị mấu DDM với hàm lượng lipid thấp.Tuy nhiên kết quả cũng gợi ý khả năng định lượng tương đối chính xác PL bằng cách kết hợp SPE và TLC.

Kiểm tra bằng TLC cũng cho thấy, quy trình SPE của Agren không lặp lại được đối với mẫu 
lipid chiết từ DDM (hình 4), và cũng chưa phát hiện được nguyên nhân. Chúng tôi không loại trừ khả năng có một số chi tiết trong quy trình, trong điều kiện mẫu hay điều kiện phân tích đã không được tác giả nêu đầy đủ.

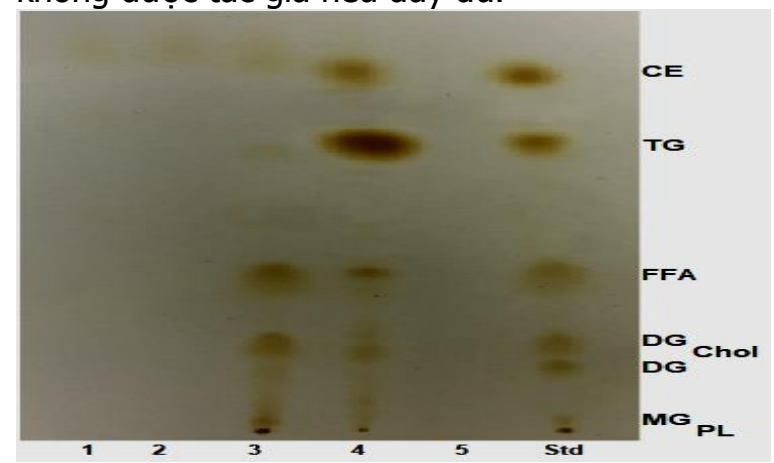

Hinh 4. Sắc đồ TLC các phân đoạn lipid tách bằng SPE theo quy trinh của Agren.

Các dải tương ứng với các phân đoạn: 1- $\mathrm{CE}$; 2- TG; 3- DG \& MG; 4- FFA; 5-PL; Std- Hốn hợp lipid chuẩn.

Hầu hết các nghiên cứu lipid bằng SPE cho đến nay tập trung vào tách các $\mathrm{PL}$ cho phân tích tiếp, bởi chúng là những thành phần có giá trị trong nhiều lĩnh vực, ở nhiều khía canh. Hơn nữa, tuy hiệu suất thu hồi PL bằng SPE không cao, song cũng là lý tưởng đối với các phương pháp điều chế thường dùng khác. Chúng tôi cũng khảo sát các quy trình SPE phổ biến để tách PL trong phân tích thành phần lipid của DDM bảo quản mẫu mô sinh học do giá trị của việc theo dõi, quản lý thành phần này trong đánh giá độ bảo toàn cấu trúc của mấu. Khi có đủ điều kiện (như thiết bị, dữ liệu tham chiếu) để nghiên cứu sâu thêm về các PL trong dung

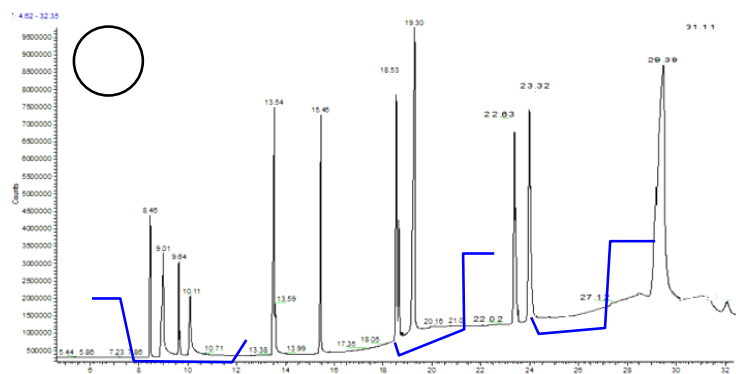

dịch, nên lựa chọn phương pháp SPE theo quy trình của Kaluzny để chuẩn bị mẫu.

Kết quả này củng cố cho định hướng sử dụng các kỹ thuật hiện đại như GC,HPLC để phân tích chuyên sâu, kết hợp TLC để định tính sơ bộ và chuẩn bị mẫu. Chúng tôi cũng đã phân tích tiếp bằng GC các phân đoạn lipid đã tách ở trên.

Các cột mao quản gắn pha tĩnh phân cực trung bình như VF-5ht có khả năng tách tốt các lớp lipid, kể cả TGtheo mức độ không bão hòa cũng như theo kích thước của các chuổi acyl béo trong phân tử. Đối với mẫu chuẩn đơn, GC hoàn toàn tách được các lipid phân cực nhẹ đến trung bình như $D G, M G$, cholesterol và $P L$ (hình $5 A$ ). Tuy nhiên, với các mẫu giàu $D G, M G$ thì việc nhận diên trên sắc đồ $\mathrm{GC}$ rất khó khăn. Bởi khi đó các $M G$ và $D G$ với thành phần acid béo khác nhau (12 - 20 carbon) tạo thành một dãy peak tiếp nối nhau. Trong đó $M G$ với acid béo mạch ngắn (C12) giải ra sát với FFA mạch dài (như C20, C22). Khoảng cách giữa các vùng $M G$, DG và TG trên sắc đồ là khá hẹp. Ở đây chúng tôi cũng thấy $\mathrm{PL}, \mathrm{CE}$ được giải ra trong vùng giữa $M G$ và $D G$ (hình $5 B$ ). Thực tế này cho thấy, để phát hiện được các lipid cấu trúc như PL và các cholesterol, cần có một phương pháp tách sơ bộ - như SPE hay TLC, trước khi phân tích bằng công cụ hiện đại (GC, HPLC).

Kết quả GC phù hợp với kiểm tra bằng TLC và cho thây, SPE không tách được tốt tất cả các thành phần lipid của mấu chiết từ DDM (hình 6). Như vậy, cùng với việc không thể đọc trực tiếp các kết quả phân tích, theo chúng tôi SPE không phù hợp với mục đích định tính nhanh các thành phần lipid trong phân tích DDM.

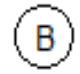

Hình 5. Các thành phần lipid phân tích bằng GC trên cột VF-5ht. A- Lipid chuẩn [FFA (C16:0,16:1,18:0, 18:1); MG (C12); CE: cholesteryl octadecanoat; $P L$ (PC, PE); DG: dilaurin và dimyristin; TG: tristearin]; B- Lipid chiết từ một mẫu DDM bảo quản mô sinh họ.

Bảng 2. Tỷ lệ các lớp lipid cứa các phân đoạn chiết pha rắn theo phương pháp Kaluzny

\begin{tabular}{|c|c|c|c|c|c|c|c|}
\hline \multirow{2}{*}{ Phân đoạn } & \multicolumn{5}{|c|}{ Tý lệ (\%, theo diện tích peak) của các lớp lipid trong phân đọan } \\
\cline { 2 - 7 } & $\mathrm{FFA}$ & $\mathrm{CE}$ & $\mathrm{PL}$ & $\mathrm{TG}$ & $\mathrm{Ch}$ & $\mathrm{DG}$ & $\mathrm{MG}$ \\
\hline $\mathrm{B}(\mathrm{FFA})$ & $\mathbf{8 2 , 1 4}$ & 1,03 & 5,28 & & - & 10,55 & 1,0 \\
\hline $\mathrm{C}(\mathrm{CE})$ & 3,71 & $\mathbf{2 0 , 5 7}$ & 12,33 & 63,39 & - & - & - \\
\hline
\end{tabular}


VIETNAM MEDICAL JOURNAL N01 - SEPTEMBER - 2021

\begin{tabular}{|c|c|c|c|c|c|c|c|}
\hline D (PL) & - & - & $\mathbf{1 0 0}$ & - & - & - & - \\
\hline E (TG) & 5,05 & 3,15 & 3,56 & $\mathbf{7 8 , 2 4}$ & 2,05 & 1,39 & 6,56 \\
\hline F(Ch) & & 5,69 & & 15,3 & $\mathbf{7 5 , 2 5}$ & 3,76 & \\
\hline G (DG) & 5 & - & & 20,56 & - & $\mathbf{5 3 , 9 4}$ & 20,5 \\
\hline H (MG) & - & - & 10 & - & - & 1,15 & $\mathbf{8 8 , 8 5}$ \\
\hline
\end{tabular}

Kết quả kiểm tra bằng GC cho thấy có thể áp dụng quy trình SPE theo Kaluzny để chuẩn bị mẫu do tăng được tỷ lệ thành phần đích trong các phân đoạn, tạo thuận tiện cho nhận diện khi phân tích GC hay HPLC. Trong đó phân đoạn PL đạt mức tinh sạch sắc ký. TLC cho thấy khả năng tách chọn lọc tốt hơn hẳn $\mathrm{SPE}$, và mặc dù có một số nhược điểm song cùng với tính đơn giản, nó vẫn là phương pháp được tiếp tục phát triển và lựa chọn rộng rãi trong phân tích. Qua kiểm tra GC, thâyphương pháp SPE của Agren cũng thu được PL tinh sạch, song hiệu quả thấp hơn phương pháp của Kaluzny.

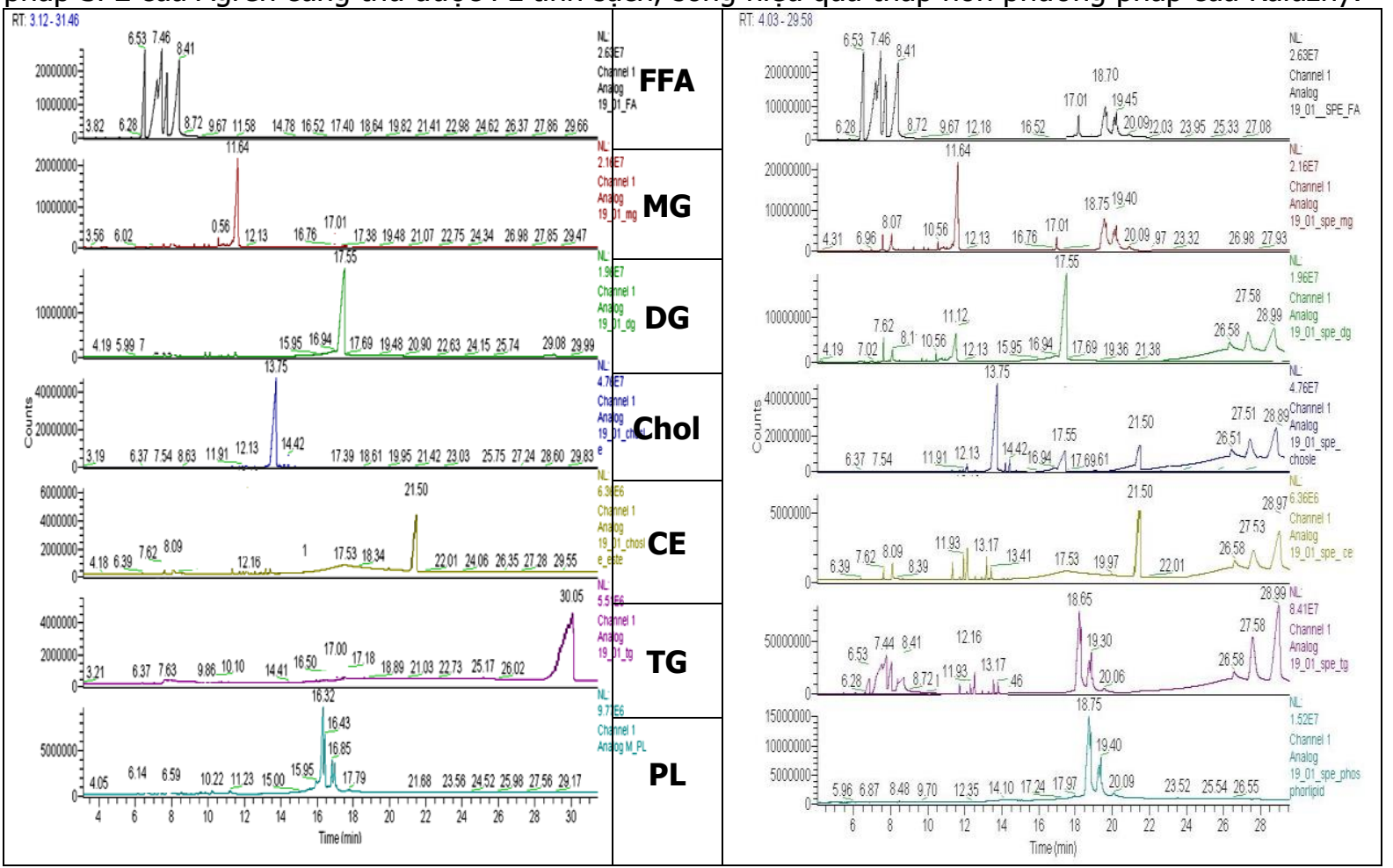

Hình 6. Sắc đồ GC các phân đoạn lipid chuẩn (chiết lại từ DDM), đã tách bằng TLC (bên trái), và bằng SPE theo phương pháp của Kaluzny(bên phải)

3.3. Phân tích thành phần lipiód trong dung dịch muối bảo quản mấu mố sinh hoc

Theo phương pháp TLC lựa chọn, đã tách được khá tốt các thành phần lipidtrong các mẫu DDM đã sử dụng.Trên bản TLC có thể đánh giá sơ bô các thành phần lipid cấu trúc như $\mathrm{PL}$, cholesterol và $\mathrm{CE}$. Thành phần FFA có hàm lượng nhỏ, phản ánh phần nào sự thoái biến của lipid trong DDM. Ngoài ra cũng thấy có một số thành phần chưa xác định được, phù hợp với kết quả phân tích GC. Như vậy, TLC theo phương án lựa chọn cho phép đánh giá định tính nhanh và có thể định lượng khi có máy đo thích hợp.

Như vậy chúng tôi đã khảo sát đánh giá được hiệu quả, đặc điểm tách thành phần lipid trong dung dịch có nồng độ muối cao bằng phương pháp chiết pha rắn và sắc ký lớp mỏng theo một số phương án, từ đó chọn được quy trình tách lớp phù hợp cho mẫu dung dịch muối. Các phương pháp khai triển một bước đều tách được các lipid trong mẫu chuẩn (hình 2). Thứ tự tách ra của các lớp theo hướng khai triển giống như quy trình của các tác giả nước ngoài. Một số đặc điểm tách có khác ở hai phương pháp sau gồm: MG di chuyển rất ít (tương tự phương pháp của Ruiz), CE di chuyển nhiều hơn. Các đặc điểm này phù hợp với tính chất của hỗn hợp dung môi sử dụng.

\section{KẾT LUẬN}

Qua khảo sát, chúng tôi thấy quy trình TLC một bước với hỗn hợp dung môi $B\left(C_{6}\right.$-DEEmethanol-acid acetic; $90: 20: 3: 2$ ) là đơn giản và 
tách khá tốt các lớp lipid chuẩn đơn cũng như hỗn hợp. Trường hợp cần tách thêm các thành phần MG, DG có thể khai triển thêm một bước với hỗn hợp chứa dung môi phân cực, chúng tôi đã thu được kết quả có cải thiện khi khai triển thêm một bước bằng $\mathrm{C}_{6}$-DEE-aceton (60:40:5) đến $12 \mathrm{~cm}$, cho phép tách các thành phần lipid một cách chọn lọc từ lipid toàn phần chiết từ DDM với độ chọn lọc, độ lặp lại cao, kết quả ổn định vàtách tốt các lớp lipid: Phospholipid; monoglycerid; diglycerid; cholesterol; acid béo tự do; triglycerid; cholesterol esthercó Rf lần lượt là : 0 ; $(0,0-0,03)$; $(0,11-0,28) ;(0,12-0,20)$; $(0,33-0,52) ;(0,66-0,75) ;(0,80-0,90)$. Các lớp lipid từ bản TLC cũng thu lại được khá đơn giản cho phân tích tiếp.Có thể dễ dàng thu các phân đoạn lipid sau khi tách bằng sắc ký lớp mỏng để tạo thuận lợi cho phân tích sâu thêm.

\section{TÀI LIÊU THAM KHẢO}

1. Nguyễn Danh Khánh (2021), Đánh giá trạng thái lipid trong mô mõ dưới da ướp bảo quản và đề xuất giải pháp nhằm bảo tồn lipid trong quá trình giữ gin lâu dài thi thể ướp phục vụ thăm viếng, Đề tài cấp Bộ, mã số KYBL-18-01.
2. Nguyễn Hồng Minh (1995), Xác định lipid trong dung dịch ướp bảo quản bằng phương pháp sắc ký lớp mỏng, Đề tài nhánh thuộc đề tài độc lập cấp Nhà nước, mã số KYĐL - 96 - 01.

3. Agren J. J., Julkunen A., Penttila I. (1992), Rapid separation of serum lipids for fatty acid analysis by a single aminopropyl column. J. Lipid Res. 33(12): 1871 - 6.

4. Kaluzny M. A., Duncan L. A., MerrittM. V., Epps D. E. (1985), Rapid separation of lipid classes in high yield and purity using bonded phase columns. Journal of lipid research, 26(1), 135-140.

5. Kupke I. R. And Zeugner S. (1978), Quantitative high-performance thin-layer chromatography of lipids in plasma and liver homogenates after direct application of $0.5-\mu l$ samples to the silica-gel layer. Journal of Chromatography B: Biomedical Sciences and Applications, 146(2), 261-271.

6. Ruiz J. I., Ochoa B. (1997), Quantification in the subnanomolar range of phospholipids and neutral lipids by monodimensional thin-layer chromatography and image analysis. J. Lipid Res., 38(7): 1482 - 9.

7. White T., Bursten S., Federighi D., Lewis R. A. (1998), Nudelman, E.High-resolution separation and quantification of neutral lipid andphospholipid species in mammalian cells and sera by multionedimensionalthin-layer chromatography. Anal. Biochem.258: 109 - 117 .

\section{CAN THIỆP NộI MACH ĐIỀU TRI HộI CHỨNG “KẸP HẠT DẺ” (NUTCRACKER) TẠI BÊ̂NH VIÊ̂N HỮU NGHI VIỆT ĐỨC: THÔNG BÁO HAI CA LÂM SÀNG}

\section{TÓM TẮT}

Hội chứng "kẹp hạt dẻ" (Nutcracker), do tĩnh mạch thận trái bị kẹp bất thường giữa động mạch mạc treo tràng trên và động mạch chủ bụng - là một hội chứng hiếm gặp biểu hiện lâm sàng nghèo nàn và thường được chân đoán khá muộn sau khi đã loại trừ các nguyên nhân khác. Việc chẩn đoán xác định bệnh dựa vào siêu âm Doppler và chụp cắt lớp vi tính ổ bụng. Có nhiều giải pháp điều trị khác nhau tùy thuộc vào đặc điểm bệnh nhân và mức độ nghiêm trọng của triệu chứng, trong đó can thiệp nội mạch là phương pháp ít xâm lấn và có hiệu quả cao. Báo cáo trình bày hai ca bệnh hẹp tĩnh mạch thận trái, được chẩn đoán là hội chứng Nutcracker, và được điêuu trị thành công

\footnotetext{
${ }^{1}$ Bệnh viện Hữu Nghị Việt Đức,

${ }^{2}$ Trường đại học Y Hà Nội

Chịu trách nhiệm chính: Phùng Duy Hồng Sơn

Email: hongsony81@yahoo.com

Ngày nhận bài: 11.6.2021

Ngày phản biện khoa học: 9.8.2021

Ngày duyệt bài: 16.8.2021
}

\section{Lê Nhật Tiên ${ }^{1,2}$, Nguyễn Minh Trí2, Nguyễn Hữu Ước ${ }^{1,2}$, Phùng Duy Hồng Sơn ${ }^{1,2}$ \\ bằng can thiệp nội mạch đặt stent tĩnh mạch thận trái tại Trung tâm Tim mạch và Lồng ngực bệnh viện Hữu nghị Việt Đức. \\ Tư khóa: Hội chứng Nutcracker, can thiệp nội mạch, Việt Đức \\ SUMMARY \\ VASCULAR INTERVENTION IN \\ NUTCRACKER SYNDROME AT VIET DUC UNIVERSITY HOSPITAL - ON THE OCCASION OF TWO CASES}

Nutcracker syndrome is rare, results most commonly from the compression of the left renal vein between the abdominal aorta and superior mesenteric artery, although other variants exist, that is usually lately diagnosed when other causes have been ruled out. The disease was diagnosed based on Doppler ultrasound and abdominal computed tomography. Treatment can vary, depending on patient characteristics and the severity of the symtoms. Endovascular intervention is a minimally invasive method with very good results. This presented report based on two cases with Nutcracker syndrome and succesfully treated by endovascular intervention with 